\title{
Proyección y responsabilidad social en las facultades de Derecho
}

\author{
IVÁN ORTIZ SÁNCHEZ*
}

\begin{abstract}
SUMARIO: INTRODUCCIÓN.- I. NORMAS UNIVERSITARIAS.- II. RESPONSABILIDAD SOCIAL UNIVERSITARIA.- III. RELACIÓN PROYECCIÓN SOCIAL Y RESPONSABILIDAD SOCIAL.- IV. RESPONSABILIDAD Y PROYECCIÓN SOCIAL EN LA FACULTAD DE DERECHO PUCP.- V. PROYECCIÓN Y RESPONSABILIDAD SOCIAL EN LATINOAMÉRICA.
\end{abstract}

\section{NTRODUCCIÓN}

En la dinámica latinoamericana y nacional, se han venido utilizando indistintamente los términos «proyección social», «extensión universitaria», «extensión interactiva», «cooperación popular universitaria» ${ }^{1} \mathrm{y}$ «labor de extensión». En Europa, se ha acuñado «cooperación universitaria al desarrollo» ${ }^{2}$ y, en la última década, se ha incorporado la visión de «responsabilidad social universitaria» o RSU.

Estos términos sirven para afirmar un eje esencial: el vínculo o la relación entre universidad y sociedad o comunidad ${ }^{3}$. Con el tiempo, este vínculo o esta relación, así como las estrategias de acción, han ido cambiando o transformándose. En ese sentido, la universidad tiene la función de colaborar con el desarrollo de la sociedad al darle su saber y su conocimiento.

La reforma de Córdoba, en Argentina (1918) ${ }^{4}$, puso de manifiesto la necesidad de que las universidades, además de cumplir su rol de formación académica, de investigación, adquiriesen un compromiso con la sociedad. Las universidades en América Latina incorporaron a sus funciones fundamentales la proyección social y la extensión universitaria, a raíz

\footnotetext{
* Es profesor ordinario del Departamento Académico de Derecho PUCP, profesor-coordinador de Proyección Social - PROSODE, y del curso de Derecho Urbanístico. El presente trabajo es una adaptación de la ponencia presentada en la mesa de Responsabilidad Social del Abogado durante el Seminario Internacional sobre Enseñanza del Derecho, realizado el 9, 10 y 11 de agosto de 2010 en la Facultad de Derecho PUCP. El autor dedica las siguientes palabras: «A mi amada Alicia. Mi cónyuge, madre de mis hijos y compañera, por su amor, comprensión y apoyo permanente que permitió inclusive participar como expositor del seminario internacional sobre Enseñanza del Derecho el día de su cumpleaños, el 11 de agosto de 2010».

1 En el seno del diálogo y de debate del evento arriba mencionado, el doctor Lorenzo Zolezzi Ibárcena mencionó que los años sesenta en el Perú se empleaba el término «cooperación popular universitaria".

2 Universidad Pública de Navarra. “Cooperación universitaria al desarrollo». Jornada de Trabajo del Consejo Social de la Universidad Pública de Navarra. Pamplona, 2003.

3 Patricia Ruiz Bravo se refiere al vínculo universidad-comunidad en una entrevista realizada en la revista Derecho PUCP, N 64, Lima, 2010.

4 Dirección Académica de Responsabilidad Social - DARS. Trasformemos la universidad peruana. Enfoque de la responsabilidad social universitaria en la PUCP. Lima: PUCP, 2009, p. 11.
} 
del debate sobre el sentido social de la universidad, despreocupada hasta entonces de la realidad y de sus problemas.

En este contexto, quiero compartir algunas reflexiones sobre la proyección y la responsabilidad social universitaria a partir del marco normativo peruano y desde las perspectivas del aprendizaje en nuestra universidad, con especial énfasis en las facultades de Derecho.

\section{NORMAS UNIVERSITARIAS}

En el marco legal peruano ${ }^{5}$, se establece que las universidades «se dedican al estudio, la investigación, la educación y la difusión del saber y la cultura, y a su extensión y proyección social $»^{6}$. Un segundo elemento son los principios de la universidad ${ }^{7}$, entre los que cabe destacar «la búsqueda de la verdad, la afirmación de los valores y el servicio a la comunidad». Un tercer elemento lo constituyen los fines de la universidad ${ }^{8}$.

Se establecen cuatro finalidades, pero queremos destacar dos de ellas:

[...] de un lado, formar humanistas, científicos y profesionales de alta calidad académica, de acuerdo con las necesidades del país, desarrollar en sus miembros los valores éticos y cívicos, las actitudes de responsabilidad y solidaridad social y el conocimiento de la realidad nacional, así como la necesidad de la integración nacional, latinoamericana y universal y, de otro lado, extender su acción y sus servicios a la comunidad y promover su desarrollo integral.

Por último, nuestra normativa establece ${ }^{9}$ que:

[...] las universidades extienden su acción educativa en favor de quienes no son estudiantes regulares; en tal sentido, organizan actividades de promoción y difusión de cultura general y estudios de carácter profesional, que pueden ser gratuitos o no, y que pueden conducir a una certificación [...] Prestan servicios profesionales en beneficio de la sociedad y regulan estas acciones en su Estatuto de acuerdo con sus posibilidades y las necesidades del país con preferencia por las regiones que correspondan a su zona de influencia.

A partir de la revisión de nuestro marco legal universitario podemos afirmar que las universidades cuentan con tres elementos o fines que construyen su esencia como organización:

- la docencia;

- la investigación; y

- la proyección y extensión social. 
De otro lado, este vínculo entre universidad y sociedad —es decir, la proyección social — se desarrolla a través de los siguientes componentes:

1. formar de acuerdo a las necesidades del país (desarrollar);

2. prestar servicios;

3. promover el desarrollo integral de la comunidad;

4. desarrollar valores éticos y cívicos; y

5. desarrollar actitudes de responsabilidad y solidaridad social.

Por último, se menciona la responsabilidad social como una actitud que las universidades deben promover y desarrollar en sus estudiantes.

\section{RESPONSABILIDAD SOCIAL UNIVERSITARIA}

Proviene de la Responsabilidad social empresarial y es entendida ${ }^{10}$ como un enfoque de gestión que promueve las buenas prácticas tanto en la administración interna de la universidad como en su vínculo con la sociedad. Está éticamente orientada en términos del cuidado de los impactos que el ejercicio de la vida universitaria genera sobre grupos internos y externos. En este contexto, nuestra universidad ha desarrollado su propia definición y modelo teórico ${ }^{11}$ :

Aplicada a la universidad, la responsabilidad social permite reformular el compromiso social universitario hacia una mayor pertinencia e integración de distintas funciones. Así la responsabilidad social universitaria (RSU) procura alinear los cuatros procesos universitarios básicos de gestión, formación, investigación y extensión con las demandas científicas, profesionales y educativas que requiere un desarrollo local y global mas justo y sostenible.

Constituye un enfoque transversal que cruza cuatro procesos o funciones universitarias básicas ${ }^{12}$ :

a. gestión interna (impacto organizacional);

b. docencia (impactos educativos);

c. investigación para el desarrollo (impactos cognitivos y epistemológicos); y

d. extensión (impactos sociales). 
Este último proceso hace referencia a los vínculos de la universidad con actores externos, como promotora del desarrollo humano sostenible. Vallaeys ${ }^{13}$ también lo denomina «proyección social».

\section{RELACIÓN ENTRE PROYECCIÓN SOCIAL Y RESPONSABILIDAD SOCIAL}

Aquí hemos encontrado una posición que contrapone proyección social y extensión universitaria con responsabilidad social universitaria ${ }^{14}$ :

[...] en la primera la universidad se vincula con la sociedad desde un enfoque unidireccional e influye en ella sin fomentar un vinculo reciproco. Desde este enfoque domina la buena voluntad de los miembros de la universidad comprometidos con el aporte a la solución de las necesidades sociales de un modo desinteresado. Las limitaciones de este modelo se han venido planteando en dos sentidos. En primer lugar, se han cuestionado la sostenibilidad y profundización de sus aportes, así como los impactos de retroalimentación que dichas experiencias pudieran significar para la universidad. En segundo lugar, este modelo se ha visto incapaz de atender las crecientes exigencias y demandas que se plantean a las universidades para contribuir con los requerimientos del desarrollo.

En este contexto, la RSU representa un enfoque renovado del compromiso social de la universidad. Se centra en la relación de mutuo beneficio entre ella y los actores externos. La idea es que profesores y alumnos participen de procesos de aprendizaje que formen parte de intervenciones universitarias bajo el enfoque del desarrollo humano sostenible. El modelo RSU supone, asimismo, que las mallas curriculares y las líneas de investigación curricular sean sensibles y se retroalimenten a partir de dichas intervenciones.

De otro lado, existe otra posición ${ }^{15}$ que afirma que la responsabilidad social surge en referencia por la preocupación de las empresas en mejorar su imagen, ya que han generado ganancias pero también impactos negativos en el medio ambiente. La responsabilidad social guarda una relación de obligación con la comunidad frente a dicho impacto. Es una especie de contraprestación. La proyección social universitaria es, en cambio, no obligatoria sino voluntaria; en ella existe una relación de solidaridad, no de reciprocidad ni contraprestación. Aquí cabe plantearse si la naturaleza de la universidad es similar a la de una empresa.

13 VALLAEYS, François. «La responsabilidad social de la Universidad». En Palestra, portal de asuntos públicos.

14 DARS. Transformemos la universidad peruana... Ob. cit., pp. 11-12.

15 PEÑA, Antonio. «La proyección social y las clínicas jurídicas en la formación de abogados. Uso en el acceso a la justicia y defensa de los derechos humanos". Video conferencia de Diálogo Global realizada el 11 de octubre de 2007 y organizada por PROSODE, Facultad de Derecho PUCP. En $<$ http://blog.pucp.edu.pe/item/16305/dialogo-global-la-proyeccion-social-y-las-clinicas-juridicas-enla-formacion-de-abogados-uso-en-el-acceso-a-la-justicia-y-defensa-de-los-derechos-humanos>. 
Por último, según lo entendió el primer Claustro Pleno de nuestra Facultad de Derecho ${ }^{16}$, la proyección social:

- Representa un eje esencial de la formación universitaria. Genera consecuencias no solo en los estudiantes sino en el quehacer universitario y dinamiza su desarrollo.

- Se comprende como una metodología de enseñanza.

- Debe cruzar todas las materias y tener lugar durante la mayor parte de la carrera de Derecho.

- Debe representar una acción institucional de la universidad.

Considero que la perspectiva de proyección social aplicada en el curso PROSODE de la Facultad de Derecho es similar al enfoque de responsabilidad social universitaria, aun cuando tienen denominaciones diferentes.

Debemos analizar también nuestra legislación universitaria, que afirma como elementos definitorios la docencia, la investigación y la proyección social o extensión universitaria, mas no la responsabilidad social. A partir de esta última perspectiva, opino que debemos trazar una línea de articulación y de complemento: el enfoque de gestión RSU es muy importante, pues cruza todos los elementos de una universidad (docencia, investigación, proyección social y organización interna) de forma transversal y genera una mirada de retorno en aprendizaje de la acción en la comunidad. Pero es un enfoque de gestión que no debe anular o desaparecer la proyección social, sino que debe dotarlo de un nuevo impulso y de una fuerza que lo trascienda para cruzar todo el quehacer universitario en forma integral.

Es importante precisar que nuestra universidad optó por un enfoque de responsabilidad social universitaria al transformar la Dirección Académica de Proyección Social y Extensión Universitaria (DAPSEU) en la Dirección Académica de Responsabilidad Social (DARS) y, dentro de ese marco, generó una serie de incentivos para promoverla en profesores y estudiantes. Entre estos se cuentan:

- premios de responsabilidad social a los profesores de la universidad que realicen acciones de RSU;

- fondos concursables de responsabilidad social universitaria a iniciativas de formación y proyectos de desarrollo; y

- fondos para investigaciones orientadas hacia el desarrollo.

16 «Informe de la Comisión de Proyección Social del Claustro Pleno». Este informe de 1991 fue presidido por el doctor Juan Luis Avendaño en la Facultad de Derecho de la PUCP y publicado en Proyección social a través del Derecho y PROSODE. 15 años proyectando el Derecho a la comunidad. Lima: PUCP, 2006, pp. 189-191. 


\section{RESPONSABILIDAD Y PROYECCIÓN SOCIALEN LA FACULTAD DE DERECHO}

Si tuviéramos que hacer un recuento de la proyección/ responsabilidad social en la Facultad de Derecho hace veintiún años, podríamos centrarnos en un actor principal o único en nuestra Facultad: el Taller de Derecho ${ }^{17}$. Este operaba en cuatro áreas: consultorios jurídicos gratuitos en Carabayllo y luego en Barranco, escuela de Derecho, diagnóstico y capacitación en Puente Piedra ${ }^{18}$ y área laboral. No había más.

\section{IV.1. PROSODE}

Luego del claustro pleno se aprueba que la proyección social es esencial en la formación del abogado y se crea, en 1991, el programa de Proyección Social de Derecho, PROSODE. Así se institucionaliza la proyección social en la Facultad de Derecho de la PUCP a través de un curso electivo de tres créditos.

Su objetivo fue y es, de un lado, articular el aprendizaje integral de conocimiento, habilidades y valores de estudiantes; y, de otro, contribuir a mejora la calidad de vida al ofrecer servicios jurídicos gratuitos a la comunidad para facilitar su acceso a la justicia, la defensa derechos humanos y la formación ciudadana.

El curso PROSODE se desarrolla a través de las siguientes áreas:

- Asesoría legal. Consiste en consultorías y seguimientos de casos en cinco consultorios jurídicos gratuitos. El alumno debe asumir el rol de abogado en casos reales, lo cual constituye un reto personal y académico. En estos consultorios, los estudiantes establecen contacto con casos concretos, presentados en forma directa por los propios involucrados; ello motiva que el estudiante participe directamente en la atención y en el seguimiento judicial de los procesos. Los alumnos tienen la oportunidad de iniciar su labor de futuros abogados, pues deben conocer el caso en su aspecto jurídico/legal y diseñar estrategias de defensa. Todo esto contribuye a que la justicia sea un bien accesible a las personas. Se trata de una clínica jurídica de interés privado o de pobres.

- Educación legal. El programa de educación legal está dirigido a fortalecer la formación ciudadana y la defensa y la promoción de los derechos de niños, niñas y adolescentes. En los centros educativos, el estudiante de PROSODE transmite sus conocimientos de Derecho y abarca una gama amplia de temas: derechos de niños y adolescentes, formación ciudadanía, entre otros. Ade-

17 «Informe de Propuesta de la Comisión de Proyección Social al I Claustro Pleno de Derecho PUCP», 1989. p. 8.

18 El autor tuvo la oportunidad de participar en el Taller de Derecho en el equipo de Diagnóstico y Capacitación de La Ensenada y Puente Piedra, zonas de extrema pobreza de Lima. Además, formó parte del equipo de Uso Alternativo del Derecho. 
más, experimenta las problemáticas que viven y sufren personas individuales e integrantes de una familia. Se trata de un contacto muy productivo entre jóvenes, donde los temas de Derecho se convierten en catalizadores para la reflexión y la conciencia del ejercicio de sus derechos. En Estados Unidos, este programa es conocido como street law o school law.

- Difusión legal. Consiste en la preparación, la producción y la emisión de programas socio-jurídicos difundidos a nivel nacional, una vez por semana, en una emisora radial. Se forman equipos de estudiantes para que aprendan a identificar los grandes problemas legales que aquejan a la población y así, a partir de su estudio y sus investigaciones, elaboren un programa radial, difundan los derechos humanos y el cumplimiento de obligaciones y absuelvan consultas al aire.

- Penal-penitenciario ${ }^{19}$. Se realiza un trabajo legal en el penal San Pedro, ubicado en San Juan de Lurigancho. Allí se asume la defensa de internos de escasos recursos económicos y se pone en práctica la vocación de servicio y los conocimientos en Derecho penal. Se visitan establecimientos penitenciarios, juzgados y salas para conocer la realidad penitenciaria nacional y del sistema de justicia penal. Es una clínica legal en cárceles.

Para apreciar el impacto del curso en los estudiantes, es importante detallar las conclusiones ${ }^{20}$ de los talleres de evaluación de los estudiantes del curso de PROSODE de los últimos años, donde se da cuenta del retorno en aprendizaje:

\begin{tabular}{|l|l|l|}
\hline \multicolumn{1}{|c|}{ Asesoría legal } & \multicolumn{1}{|c|}{ Educación legal } & \multicolumn{1}{c|}{ Difusión legal } \\
\hline $\begin{array}{l}\text { Permite poner en práctica } \\
\text { conocimientos jurídicos } \\
\text { adquiridos. }\end{array}$ & $\begin{array}{l}\text { Despierta el aspecto so- } \\
\text { cial y humano del estu- } \\
\text { diante. }\end{array}$ & $\begin{array}{l}\text { Contribuye con nuestra co- } \\
\text { munidad. }\end{array}$ \\
\hline $\begin{array}{l}\text { Permite establecer un } \\
\text { contacto directo con la } \\
\text { realidad social. }\end{array}$ & $\begin{array}{l}\text { Refuerza, mejora y aplica } \\
\text { conocimientos académi- } \\
\text { cos. }\end{array}$ & $\begin{array}{l}\text { Ayuda a conocer realidades } \\
\text { distintas. }\end{array}$ \\
\hline $\begin{array}{l}\text { Genera responsabilidad y y } \\
\text { compromiso social. }\end{array}$ & $\begin{array}{l}\text { Forma mejores ciuda- } \\
\text { danos con conciencia } \\
\text { cívica. }\end{array}$ & \begin{tabular}{l} 
Refuerza jurídicos. \\
\hline
\end{tabular} \\
\hline
\end{tabular}

19 Este trabajo se realiza gracias a un convenio con el Instituto Nacional Penitenciario - INPE ideado por el profesor Juan Luis Avendaño, con el apoyo de Leonardo Caparrós, abogado por la Pontificia Universidad Católica del Perú y ex presidente del INPE, y gracias al compromiso y la coordinación del doctor Iván Meini Méndez, coordinador del Área Penal del Departamento Académico de Derecho, impulsado en el marco de las celebraciones de los noventa años de la Facultad de Derecho.

20 ZUTA, Erika e Iván ORTIZ. «Participación protagónica de los estudiantes de la Facultad de Derecho y el impacto del aprendizaje-servicio en la calidad de sus aprendizajes». En María Nieves Tapia (editora). Actas del décimo primer Seminario Internacional Aprendizaje y Servicio Solidario. Buenos Aires, 2009. 


\begin{tabular}{|l|l|l|}
\hline $\begin{array}{l}\text { Posibilita el aprendizaje } \\
\text { práctico de diversos temas } \\
\text { del Derecho. }\end{array}$ & $\begin{array}{l}\text { Promueve el desarrollo } \\
\text { de habilidades comuni- } \\
\text { cativas (lenguaje, habla } \\
\text { en público, dominio de } \\
\text { escena). }\end{array}$ & Incentiva la investigación \\
\hline $\begin{array}{l}\text { Desarrolla habilidades: } \\
\text { investigación, interacción } \\
\text { con la realidad y planifica- } \\
\text { ción de estrategias. }\end{array}$ & $\begin{array}{l}\text { Permite interrelacionar- } \\
\text { se con niños y adoles- } \\
\text { centes. }\end{array}$ & $\begin{array}{l}\text { Permite explicar los } \\
\text { conocimientos } \\
\text { manera precisa y clara. }\end{array}$ \\
\hline $\begin{array}{l}\text { Permite al alumno actuar } \\
\text { como abogado al absolver } \\
\text { consultas. }\end{array}$ & $\begin{array}{l}\text { Se transmite informa- } \\
\text { ción sobre sus derechos } \\
\text { a niños y adolescentes. }\end{array}$ & $\begin{array}{l}\text { Fomenta la creatividad en } \\
\text { la elaboración del guión }\end{array}$ \\
\hline $\begin{array}{l}\text { Permite agilizar el razona- } \\
\text { miento jurídico. }\end{array}$ & $\begin{array}{l}\text { Fomenta la creatividad } \\
\text { de los estudiantes al es- } \\
\text { tructurar sus clases. }\end{array}$ & $\begin{array}{l}\text { Promueve el trabajo en } \\
\text { equipo. }\end{array}$ \\
\hline $\begin{array}{l}\text { Buena metodología apli- } \\
\text { cada en clase. }\end{array}$ & $\begin{array}{l}\text { Reconocimiento en la } \\
\text { comunidad. }\end{array}$ & $\begin{array}{l}\text { Adecuada metodología } \\
\text { (sistema de competencias). }\end{array}$ \\
\hline $\begin{array}{l}\text { Buena disposición y orien- } \\
\text { tación de los jefes de los } \\
\text { profesores. }\end{array}$ & $\begin{array}{l}\text { Integración de padres e } \\
\text { hijos. }\end{array}$ & $\begin{array}{l}\text { Buena disposición y orien- } \\
\text { tación de los profesores. }\end{array}$ \\
\hline $\begin{array}{l}\text { Materiales y bibliografía } \\
\text { adecuados. }\end{array}$ & $\begin{array}{l}\text { Facilita el desenvolvi- } \\
\text { miento profesional. }\end{array}$ & $\begin{array}{l}\text { Contribuye con nuestra } \\
\text { comunidad. }\end{array}$ \\
\hline $\begin{array}{l}\text { Facilita elaccesodelosmás } \\
\text { necesitados a la justicia. }\end{array}$ & \begin{tabular}{l} 
de trabajar con niños. \\
\hline
\end{tabular} & \\
\hline
\end{tabular}

\section{IV.2. Otras iniciativas}

Existen otras experiencias de nuestra Facultad de Derecho a través de cursos como la Clínica Jurídica de Interés Público, el curso Derecho y Desastre, y el curso de Derecho y Desarrollo. Desde los estudiantes, se cuenta con las comisiones sociales de las revistas Themis, Ius Veritas, Derecho y Sociedad, y con las asociaciones Taller de Derecho e Iter Criminis. Para más detalles, puede revisarse el número 64 de la revista Derecho PUCP, en la que se da cuenta de las experiencias de responsabilidad social de la Facultad de Derecho.

Revisando estas iniciativas podemos afirmar que encontramos diversas y múltiples acciones de proyección y responsabilidad social universitaria en nuestra Facultad, lo que constituye una fortaleza con relación a la sociedad, pero que puede ser profundizada más. 


\section{PROYECCIÓN Y RESPONSABILIDAD SOCIAL EN LATINOAMÉRICA}

En Latinoamérica existen diversos programas, iniciativas y acciones de proyección/responsabilidad social con diversas denominaciones, metodologías, objetivos y naturalezas. Solo destacaremos algunas:

- Denominaciones. Bufete popular (México) y socorro popular (Centroamérica), clínica de asistencia legal (Puerto Rico), consultorios jurídicos (Perú y otros países) y clínica jurídica orientada a diversas áreas e intereses.

- Naturaleza. Algunos se presentan como actividades de estudiantes, actividades voluntarias u obligatorias de facultades de Derecho, así como cursos obligatorios o voluntarios.

- Metodología. Aprendizaje en la realidad, aprendizaje-servicio, aprendizaje basado en problemas o proyectos, método clínico.

\section{RETOS}

Por último, planteamos los siguientes retos para la Facultad de Derecho de la Pontificia Universidad Católica del Perú:

- articular acciones de PS/RSU dentro de una política común de la Facultad de Derecho y en forma interdisciplinaria con otras facultades;

- programar como obligatorias las experiencias de los cursos de proyección social y clínica jurídica en la malla curricular;

- articularlas a otros cursos como Razonamiento Jurídico, Ética, Destrezas y Habilidades; y

- promover diferentes perfiles del abogado, no solo desde la perspectiva litigante. 